\title{
Correspondence
}

\section{Policy tips: six more practical pointers}

Politicians are likely to dismiss the excellent 'Twenty tips for interpreting scientific claims' after a few lines, simply because these tips are not geared to their everyday needs (W. J. Sutherland et al. Nature 503, 335-337;

2013). I have some other suggestions for them.

Science is open-minded, but not empty-headed. Just because there is no definitive answer to a problem does not mean that any alternative is equally good. Not all evidence may point in one direction. Trust the weight of evidence.

Avoid 'false balance' on scientific issues. There will always be naysayers. That is fine in politics, in which everyone is entitled to their opinions, but not in science, in which one produces the evidence or holds one's peace.

Science is not decided by vote. Most scientists have little expertise outside their own fields. Let people who really understand the issues help you to evaluate the evidence.

Enlist the best advice on scientific issues. Do not rely on 'independent scholars' and thinktank policy wonks for advice and referrals. Every country has academies of science and excellent universities to consult. Steer clear of science that has not been peer reviewed. Conscientious analysis by our peers makes science work. It does not ensure certitude, but it beats the alternative.

Championing good science makes you look good. Knownothings and anti-intellectuals will try to undermine your principled support of some scientific issues. Turn their ignorance against them by showing the consequences of following their path.

Non-scientists could also consult undsci.berkeley.edu for an introduction to how science is done.

Kevin Padian Museum of

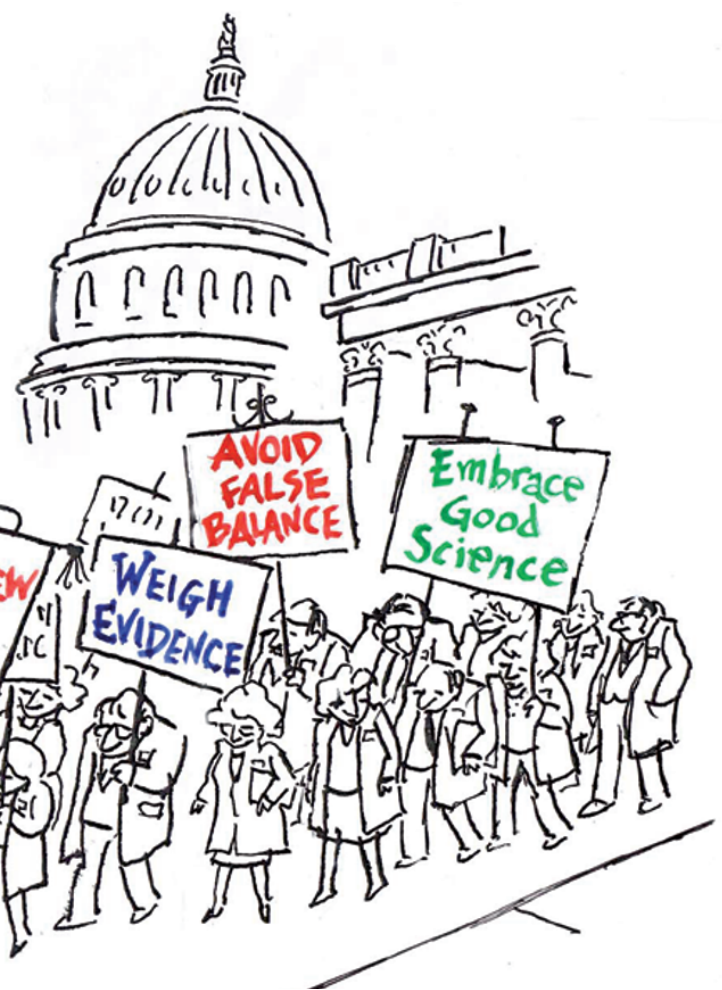

Paleontology, University of California, Berkeley, USA. kpadian@berkeley.edu

\section{Policy tips: heed risks of uncertainty}

William Sutherland and colleagues' 20 tips for nonscientists on how to interpret scientific evidence could do more harm than good in the hands of policy-makers (Nature 503, 335-337; 2013).

My experience in naturalresource management taught me that emphasizing uncertainty can lead policy-makers to dismiss evidence rather than to embrace it. On one occasion when I testified before Congress, I was told to come back only when I was $100 \%$ certain of my information. Practically speaking, this might be viewed as an excuse for political inaction.

For policy-makers, assessing the potential risks and benefits of policy action or inaction should be critical. For instance, when scientific uncertainty about the likelihood of a severe weather event is high but the consequences are potentially dire, there is a large risk in taking no protective action.

Rather than trying to teach non-scientists how to interpret uncertainty in our results, let's do a better job of helping them to understand the possible consequences of what they do or don't do.

Andrew A. Rosenberg Center for Science and Democracy, Union of Concerned Scientists, Cambridge, Massachusetts, USA.

arosenberg@ucsusa.org

\section{Life sciences lag in stringent standards}

Initiatives to ensure scientific reproducibility in life-science research (M. Bissell Nature 503, 333-334; 2013) should demand strict standards, such as the use of only authenticated cell lines or the validation of commercially available antibodies. Setting guidelines for standards brings diverse stakeholders together and fosters an environment for adherence.

Carefully devised standards have underpinned advances in industries such as engineering and technology - from Wi-Fi to building bridges. It is time for the life-sciences community to catch up.

Leonard Freedman Global Biological Standards Institute, Washington DC, USA.

lfreedman@gbsi.org

\section{EU fructose claim ignores risks}

After a long discussion within and between member states, the European Union (EU) register of authorized health claims for foodstuffs will be expanded in January 2014 to include the claim: "consumption of foods containing fructose leads to a lower blood glucose rise compared to foods containing sucrose or glucose" (see go.nature.com/tf2iov). This claim fails to mention some potentially harmful effects of fructose metabolism (see, for example, C. A. Lyssiotis and L. C. Cantley Nature 502, 181-182; 2013, and R. H. Lustig et al. Nature 482, 27-29; 2012).

As the European Food Safety Authority noted during the authorization process (see go.nature.com/dbjkxm), there is evidence that a high intake of fructose can lead to metabolic complications such as abnormal lipid levels, resistance to insulin and increased visceral adiposity.

Companies will be able to use this claim to promote sweetened foods and drinks, as long as at least $30 \%$ of the glucose or sucrose is replaced by fructose. Only stronger evidence for the risks of high fructose intake can stop this. Igor Pravst Nutrition Institute, Ljubljana, Slovenia. igor.pravst@nutris.org 